\title{
How precise can bony landmarks be determined on a CT scan
} of the knee?

\author{
J. VICTOR*, D. VAN DONINCK**, L. LABEY***, B. INNOCENTI*** \\ P. M. PARIZEL $* * * *$ and J. BELLEMANS**
}

*AZ St-Lucas, Brugge, Belgium

** Catholic University Leuven, Belgium

$* * *$ European centre for knee research, Haasrode, Belgium

**** University of Antwerp, Belgium

Key words

Knee, Imaging, Alignment, Reproducibility

Corresponding author:

J. Victor

Beukenlaan 23

8310 Brugge, Belgium

tel : +3250374423

fax: +3250376172

j.victor@skynet.be 


\section{Abstract}

The purpose of this study was to describe the intra- and inter-observer variability of the registration of bony landmarks and alignment axes on a Computed Axial Tomography (CT) scan. Six cadaver specimens were scanned. Three-dimensional surface models of the knee were created. Three observers marked anatomic surface landmarks and alignment landmarks. The intra- and inter-observer variability of the point and axis registration was performed. Mean intra-observer precision ranks around $1 \mathrm{~mm}$ for all landmarks. The intra-class correlation coefficient (ICC) for interobserver variability ranked higher than 0.98 for all landmarks. The highest recorded intra- and inter-observer variability was $1.3 \mathrm{~mm}$ and $3.5 \mathrm{~mm}$ respectively and was observed for the lateral femoral epicondyle. The lowest variability in the determination of axes was found for the femoral mechanical axis (intra-observer $0.12^{\circ}$ and inter-observer $0.19^{\circ}$ ) and for the tibial mechanical axis (respectively $0.15^{\circ}$ and $0.28^{\circ}$ ). In the horizontal plane the lowest variability was observed for the posterior condylar line of the femur (intra-observer $0.17^{\circ}$ and inter-observer $0.78^{\circ}$ ) and for the transverse axis (respectively $1.89^{\circ}$ and 2.03 ) on the tibia. This study demonstrates low intra- and inter-observer variability in the $\mathrm{CT}$ registration of landmarks that define the coordinate system of the femur and the tibia. In the femur, the horizontal plane projections of the posterior condylar line and the surgical and anatomical transepicondylar axis can be determined precisely on a CT scan, using the described methodology. In the tibia, the best result is obtained for the tibial transverse axis. 


\section{Introduction}

The use of Computed Axial Tomography (CT scan) as a medical imaging tool has widespread applications in the field of knee surgery. It is routinely used in the diagnosis and treatment of peri-articular fractures and patellofemoral pathology. In arthroplasty surgery, adoption of this technology has been slower. The CT scan is nowadays considered the premium tool for planning and evaluation of lower limb alignment $^{1}$, and this can be attributed to the development of technological applications like computer navigation and robotic surgery. These technological achievements put accurate medical imaging to the forefront of orthopedic surgery and research of the $\mathrm{knee}^{2-8}$. In the field of total knee arthroplasty (TKA), the CT scan serves different applications. Surgeons use a CT scan in a conventional way during the pre-operative stage, to plan the position of the femoral component in the horizontal plane $e^{9-11}$. In the post-operative stage, the use of a CT scan is a routine tool in the evaluation of failed $\mathrm{TKA}^{12}$, as rotational malalignment of the femoral component has been determined as a main cause of poor clinical outcome after $\mathrm{TKA}^{12-21}$. In image-based computerassisted surgery, the CT scan provides three-dimensional anatomic details $\mathrm{s}^{2-4,7}$. Novel techniques use CT-based patient-specific templating to achieve the desired alignment in TKA without the use of conventional alignment jigs. Finally, in-vivo kinematic research of the native knee relies on $\mathrm{CT}^{6}$, or $\mathrm{MRI}^{6,22}$ derived bone models. Those are used for model registration-based three-dimensional kinematic measurements, computed from sequential two-dimensional X-ray images.

In all of the above-mentioned clinical applications, surface-derived anatomical landmarks provide the link between the $\mathrm{CT}$ scan data and surgically relevant references that can be found by visualization or palpation during the operation. In 
addition, for the surgical navigation and patient-specific templating applications, the CT scan is used to define the common coordinate system, providing the surgeon the frontal, sagittal and horizontal plane of the femur and the tibia. It is fair to question the ability to accurately identify the surface-derived anatomical references and the reference points needed to provide the common coordinate system that defines the three above-mentioned clinical planes. Relatively few publications addressed this issue. Most studies concentrate on the relative position of different axes ${ }^{15-17,23-26}$. Only few evaluate intra- or inter-observer variability ${ }^{26-31}$. To our knowledge, no study has investigated a full set of surface-derived landmarks and alignment landmarks for inter- and intra-observer variability.

In order to avoid semantic confusion, the following definitions are used. Accuracy is defined as the closeness of a given measurement to the actual value for the variable considered. Precision is defined in terms of the measurement error, as the deviation of a set of repeated measurements from an arbitrary value ${ }^{32}$. As such, two observers can be very precise in their measurements (small measurements errors) but very inaccurate because of a consistent positive or negative error. Applied to this study, previous work has shown that a calibrated CT scan is a highly accurate tool. The objectives of this study were two-fold:

1. To evaluate the intra- and inter-observer precision in the locating reference points on a surface reconstruction of the femur and the tibia, based on CT scans of fresh frozen amputated leg specimens.

2. To evaluate the intra- and inter-observer precision of the corresponding axes, relevant for surgical use. 


\section{Materials and Methods}

Six unpaired fresh frozen amputated legs ( 3 right, 3 left) were analyzed, using a helical CT scan (General Electric Lightspeed VCT, Milwaukee, WI, USA). The specimens were obtained from 1 female and 5 male Caucasian subjects, aged between $78 \mathrm{y}$ and $87 \mathrm{y}$ old when they deceased. The images were obtained at $120 \mathrm{kV}$ and 450 $\mathrm{mA}$, with a slice thickness of $1.25 \mathrm{~mm}$ and a pitch of $0.5 \mathrm{~mm} / \mathrm{rev}$. Raw data were processed using a bone filter. The CT scans were analyzed using Mimics ${ }^{\circledR} 11.02$ and its MedCAD module (Materialise, Haasrode, Belgium) to create the surface reconstruction and identify the bony landmarks. Three observers participated in the study: one experienced orthopedic surgeon (JV), one medical student (DVD) and one engineer (LL). The surgeon defined the set of relevant landmarks and provided the two other observers with a definition and a brief teaching session. Afterwards, the three observers analyzed the CT scans independently. Two observers (DVD and LL) performed all analyses three times with a minimum interval of one week for obtaining intra-observer repeatability. The thresholding feature in Mimics was used to define two masks (one for the distal femur and one for the proximal tibia and fibula). Lower and higher threshold values were defined manually. The masks were then cropped to the peri-articular areas of the bones and edited to separate the different bones. Finally, the masks were converted into 3D models for identification of the anatomical landmarks.

Anatomical landmarks of the femur (Fig 1)

- Femoral Hip Centre (FHC): centre of best-fit sphere to the head of the femur

- Femoral Knee Centre (FKC): most anterior point in the middle of the femoral notch on a caudal to cranial view of the femur, aligning the hip centre with the roof of the femoral notch. 
- Femoral Medial Condyle Centre (FMCC): centre of the best-fit sphere to the medial condyle

- Femoral Lateral Condyle Centre (FLCC): centre of the best-fit sphere to the lateral condyle.

- Femoral Medial Epicondyle (FME): most anterior and distal osseous prominence over the medial aspect of the medial femoral condyle ${ }^{32}$.

- Femoral Medial Sulcus (FMS): depression on the bony surface slightly proximal and posterior to $\mathrm{FME}^{33}$.

- Femoral Lateral Epicondyle (FLE): the most anterior and distal osseous prominence over the lateral aspect of the lateral femoral condyle ${ }^{33}$.

- Femoral Trochlea Proximal (FTP): deepest point of the trochlear groove on the 3D model of the femur, aligned along the femoral mechanical axis (FMAx).

- Femoral Medial Condyle Posterior (FMCP): the most posterior point of the medial condyle on the 3D model of the femur, aligned along the FMAx.

- Femoral Lateral Condyle Posterior (FLCP): the most posterior point of the lateral condyle on the 3D model of the femur, aligned along the FMAx. Anatomical landmarks of the tibia (Fig 2)

- Tibial Ankle Centre (TAC): the centre of the best-fit circle of the tibial plafond.

- Tibial Knee Centre (TKC): the midpoint between the two tibial spines projected on the bony surface, identified by viewing the 3D model of the tibia from cranial along the tibial shaft axis.

- Tibial Medial Condyle Centre (TMCC): the centre of the best-fit circle around the edge of the cortex of the medial tibial plateau ${ }^{27}$.

- Tibial Lateral Condyle Centre (TLCC): the centre of the best-fit circle around the edge of the cortex of the lateral tibial plateau ${ }^{27}$. 
- Tibial Medial Condyle Posterior (TMCP): the most posterior point of the medial tibial plateau, on a cranial view, aligned along the tibias shaft axis.

- Tibial Lateral Condyle Posterior (TLCP): the most posterior point of the lateral tibial plateau, on a cranial view, aligned along the tibial shaft axis.

- Tibial Tubercle Anterior (TTA): the most anterior point of the tibial tuberosity, on a cranial view, aligned along the tibial shaft axis.

Consequently, we obtained seven sets of coordinates (three analyses by two of the three observers, one analysis by one observer) for the 17 landmarks in each of the six specimens. Intra- and inter-observer variability was expressed as the distance between the mean position of a landmark to the observed position of the landmark ${ }^{34}$. For intra-observer precision, the mean positions of the landmarks $\bar{P}(\bar{x}, \bar{y}, \bar{z})$ and the distances $D_{i}$ of the observed position to that mean position were defined as follows (subscripts 1,2 and 3 refer to the different observations with 1 week interval):

$$
\begin{aligned}
\bar{x} & =\frac{x_{1}+x_{2}+x_{3}}{3} \\
\bar{P}=\frac{P_{1}+P_{2}+P_{3}}{3} \rightarrow \bar{y} & =\frac{y_{1}+y_{2}+y_{3}}{3} \quad D_{i}=\left\|\bar{P}-P_{i}\right\|=\sqrt{\left(\bar{x}-x_{i}\right)^{2}+\left(\bar{y}-y_{i}\right)^{2}+\left(\bar{z}-z_{i}\right)^{2}} \\
\bar{z} & =\frac{z_{1}+z_{2}+z_{3}}{3}
\end{aligned}
$$

The mean value and standard deviation of $D_{i}$, was then calculated for each landmark as a measure of the overall intra-observer variability for that landmark.

For inter-observer precision, the mean positions of the landmarks $\overline{\bar{P}}(\overline{\bar{x}}, \overline{\bar{y}}, \overline{\bar{z}})$ were calculated using the means of the coordinates found by each observer, giving the following formulas (subscripts 1, 2 and 3 refer now to the respective observers):

$$
\begin{aligned}
\overline{\bar{x}} & =\frac{\bar{x}_{1}+\bar{x}_{2}+\bar{x}_{3}}{3} \\
\overline{\bar{P}}=\frac{\bar{P}_{1}+\bar{P}_{2}+\bar{P}_{3}}{3} \rightarrow \overline{\bar{y}} & =\frac{\bar{y}_{1}+\bar{y}_{2}+\bar{y}_{3}}{3} \quad D_{i}=\left\|\overline{\bar{P}}-\bar{P}_{i}\right\|=\sqrt{\left(\overline{\bar{x}}-\bar{x}_{i}\right)^{2}+\left(\overline{\bar{y}}-\bar{y}_{i}\right)^{2}+\left(\overline{\bar{z}}-\bar{z}_{i}\right)^{2}} \\
\overline{\bar{z}} & =\frac{\bar{z}_{1}+\bar{z}_{2}+\bar{z}_{3}}{3}
\end{aligned}
$$

Mean value and standard deviation of the three $D_{i}$ obtained from observers 1,2 and 3 were calculated for each landmark as a measure of the overall inter-observer variability for that landmark. 
To be able to discriminate between precisions along the relevant anatomical axes, a coordinate frame was defined for the femur and the tibia, based on the mean positions of the selected landmarks. For the femur, the femoral mechanical axis (FMAx) was defined as the line joining the femoral knee centre and the femoral hip centre (FHCFKC). The frontal plane was defined as the plane that contains the FMAx and is parallel to the line joining the medial and lateral centers of the femoral condyles. The horizontal axis was defined as the perpendicular line to the FMAx in the frontal plane, containing the femoral knee centre. The horizontal plane contains the horizontal axis and is perpendicular to the frontal plane. The sagittal axis was defined as the line perpendicular to the FMAx and the horizontal axis and passes through the knee centre. For the tibia the tibial mechanical axis (TMAx) was defined as the line joining the centre of the tibial plateau and the centre of the ankle (TKC-TAC). The frontal plane of the tibia was defined as the plane containing the TMAx and parallel to line joining the medial and lateral tibial condylar centre. The horizontal axis of the tibia was defined as the perpendicular line to the TMAx in the frontal plane, passing through the centre of the tibial knee centre. The horizontal plane of the tibia is perpendicular to the frontal plane and contains the tibial horizontal axis. The sagittal axis of the tibia was defined as the line perpendicular to the TMAx and the horizontal axis, passing through the tibial knee centre. All measured coordinates of all landmarks were transformed into these coordinate frames to evaluate reproducibility along the three Cartesian Axes of the bones.

In a final step, the intra- and inter-observer variation of the femoral and tibial axes was quantified, based on the mean deviation of their defining landmarks. It was assumed that the errors in the coordinates of the landmarks were independent and random and that simple error propagation estimations could therefore be used. This 
was done for the mechanical axes of femur and tibia (FMAx and TMAx) and for the axes with surgical relevance to rotational alignment, with the following definitions.

- Anatomical transepicondylar axis: FME-FLE.

- Surgical transepicondylar axis: FMS-FLE.

- Femoral posterior condylar line: FMCP-FLCP.

- Femoral transverse axis: FMCC-FLCC.

- Femoral trochlear antero-posterior axis: FKC-FTP.

- Tibial posterior condylar line: TMCP-TLCP

- Tibial transverse axis: TMCC-TLCC.

- Tibial Tubercle axis: TKC-TTA.

For the measurement of intra- and inter-observer angular differences in the rotation axes of femur and tibia, a geometrical projection on the horizontal plane of the femur and the tibia was respectively carried out.

For each of the considered landmarks positions, we evaluated the intra-class correlation coefficient (ICC) for multiple measurements by different observers on different specimens ${ }^{34}$. By definition, the ICC is evaluated according to the following formulation: $I C C=\frac{\sigma_{b}^{2}}{\sigma^{2}}$

Where the total variance of measurements by different observers is $\sigma^{2}$ on different subjects, and the variance between subjects is $\sigma_{b}^{2}$. ICC values range from 0 to 1 , indicating better agreement as the value approaches 1 . An ICC value higher than 0.75 indicates excellent agreement. The statistical analysis was performed using Matlab R2008a (The MathWorks, Natick, Massachusetts, USA). For all recorded distances and angles, mean values, maximum values and standard deviations are reported. 


\section{Results}

The magnitudes of intra-observer and inter-observer variability for each landmark are shown in Figures 3 and 4 respectively. The observed mean values, maximum values and standard deviations are displayed separately. Mean intra-observer variability for all landmarks is situated around $1 \mathrm{~mm}$ (range: $0.4 \mathrm{~mm}-1.4 \mathrm{~mm}$ ). All joint centres (FHC, FKC, TKC, TAC) and condyle centres (FLCC, FMCC, TLCC, TMCC) can be identified with a mean variability of less than $1 \mathrm{~mm}$. The femoral epicondyles and sulcus, as well as the posterior points on the tibial condyles are least reliable with mean variabilities larger than $1 \mathrm{~mm}$.

Inter-observer variability is larger than intra-observer variability and more different amongst landmarks, but is still acceptable (range: $0.3 \mathrm{~mm}-3.5 \mathrm{~mm}$ ). Again, the joint centres are most reliable with mean inter-observer variabilities of less than $1 \mathrm{~mm}$, with the exception of the tibial knee centre. The posterior points on the tibial condyles, the tibial tubercle and the femoral lateral epicondyle are least reliable with mean interobserver variabilities of more than $2 \mathrm{~mm}$.

Table 1 shows the mean and the standard deviation of the distance from observed position to the mean position for all landmarks, split along the three anatomical axes. In general, landmarks that are located on a bony surface can be identified very reliably in the direction perpendicular to the surface. The variability is usually almost twice as large in the directions tangent to the surface.

The ICC values for all defined landmarks fall in a range between 0.986 and 1 , showing that observer agreement and reliability for all landmarks is excellent. The statistical results confirm that the joint centres (with the exception of the tibial knee centre) and the posterior points on the femoral condyles are most reliable. $(\mathrm{ICC}=1)$ 
The epicondyles, the medial sulcus, the posterior points of the tibia, the tibial knee centre and the tibial tubercle have a slightly lower ranking (respective ICC values: $0.99 ; 0.99 ; 0.99 ; 0,99 ; 0,98)$

The resulting angular variation between the different axes could be computed, based on the defined landmarks.(Table 2) The mechanical axes in femur and tibia can be determined very accurately due to the reliability of the landmarks on which they are based and the large distance between the defining points. (FMAx $0.05^{\circ}$ intra- and $0.08^{\circ}$ inter-observer, TMAx $0.15^{\circ}$ and $0.28^{\circ}$ respectively). Of the axes relevant for rotational alignment, the trochlear antero-posterior axis is least reliable (mean interobserver deviation of $2^{\circ}$ ), while the posterior condylar line is most reliable with a mean inter-observer deviation of $0.5^{\circ}$. The anat TEA and surg TEA fall in between the two aforementioned axes. In the tibia, the transverse axis as defined recently by

Cobb et al. ${ }^{27}$, shows a mean intra- and inter-observer variability of respectively $1.44^{\circ}$ and $1.66^{\circ}$. The two other axes that define rotation are less reliable: posterior condylar line respectively $1.37^{\circ}$ and $3.16^{\circ}$, and the tubercle axis $1.09^{\circ}$ and $2.42^{\circ}$.

A graphical representation is shown in Figure 5.

\section{Discussion}

Amongst clinicians, the CT scan is often considered the ultimate precision tool in measuring alignment in the lower limb ${ }^{1}$. The outcome of a given procedure in terms of alignment or position is often described as a comparison to a reference value, obtained from a CT scan ${ }^{8,36,37}$. It must be emphasized that the actual reference value (plane, axis or point) remains unknown and determination of points and axes on a CT scan is subject to inter- and intra-observer variability. As appears from our results, the intra- and inter-observer variability of the landmarks that define the coordinate system 
of the femur and the tibia, is low. This is fundamental, as it is the basis for all applications of CT data in the clinical setting ${ }^{2-8}$. However, some of the study weaknesses have to be understood. First, surface modeling and landmark registration occurred in optimal circumstances on dedicated computer stations, after studying the recent anatomic literature and with anatomic drawings at hand. It is clear that this is not the real life clinical setting where surgeons often work under substantial time constraints. Also, we picked the tool that is most suited for imaging bone and providing Cartesian coordinates, the CAT scan. It is unclear whether the same accuracy could be achieved in using an MRI scan. As the cartilage contours can be defined in much greater detail on MRI scans, this tool is more suitable for patient specific templating ${ }^{38}$ and model registration-based three-dimensional kinematic measurements ${ }^{6,22}$. Because of the different qualities, with the CAT scan being better for defining the bony surface and the MRI being better for defining the cartilage surface, some research groups have used the combination of both for optimal imaging ${ }^{6}$.

Failure to obtain correct alignment in total knee arthroplasty leads to inferior results and early revisions ${ }^{12-21,39-43}$. Errors can occur at different levels: application of a wrong reference definition (e.g. the direct use of the posterior condylar line for rotational alignment of the femoral component), individual variability in the subjects (e.g. dysplasia of the lateral condyle), the radiological or surgical location of reference landmarks (e.g. locating the epicondyles), instrumental errors (e.g. mechanical play), and execution errors (e.g. fixation of cutting blocks and making of the bone cuts). This study only deals with one of those items: the precision of locating reference landmarks on a CT scan. 
Nishihara et al. ${ }^{7}$ reported the accuracy of registration in terms of position and angle to be $0.8 \mathrm{~mm}$ and 0.6 degrees of bias with $0.2 \mathrm{~mm}$ and 0.3 degrees of root-mean-square in the femur, and $0.5 \mathrm{~mm}$ and 0.4 degrees of bias with $0.2 \mathrm{~mm}$ and 0.3 degrees of root-mean-square in the tibia. The aim of this study was to determine the precision of intra- and inter-observer measurement on a CT scan in a clinically relevant setting: how reproducible is the location of relevant surface points and axes? This information can help in the development of surgical navigation algorithms, patient specific cutting blocks, and pre-operative surgical planning.

The precision in locating certain landmarks on a CT scan cannot be extrapolated to the precision of locating landmarks intra-operatively. Several authors have emphasized the important inter- and intra-observer variability in the surgical location of the femoral epicondyles $8,10,24,25,29,44$. Even in more idealized circumstances, using cadavers with or without soft tissues there is significant variability among observers ${ }^{36,}$ 37, 45-47. Yau et al. ${ }^{37}$ reported high inter-observer variability in the detection of the anatomic epicondylar axis using 5 cadavers and surgical navigation: $9^{\circ}$ of maximum error due to medial epicondyle registration error and $7^{\circ}$ due to the lateral epicondyle registration error. In a similar experiment, Stöckl et al. ${ }^{47}$ reported the inter-observer variability as a $95^{\text {th }}$ percentile of the distances between the clinical registrations, compared to the CT registrations. For the medial epicondyle, the reported distance was $14.9 \mathrm{~mm}$ in the antero-posterior direction and $18.7 \mathrm{~mm}$ in the proximal-distal direction. For the lateral epicondyle, the reported distance was $15.7 \mathrm{~mm}$ and $19 \mathrm{~mm}$ respectively. Compared to this reported variability in intra-operative landmark registration, the CT based registration proves to be superior, as shown in our results. In addition, there is clinical literature evidence that the use of a pre-operative CT scan offers opportunities to enhance surgical precision in $\mathrm{TKA}^{9-11}$, and increased use of 
this tool is to be expected in the future in an attempt to avoid outliers in post-operative alignment. Recent publications ${ }^{11,48}$ confirm the clinical trend to include pre-operative CT scans in the planning of the procedure. Knowledge of the precision of landmark allocation is mandatory to further improve surgical outcomes.

The mean intra- and inter-observer error for all landmarks that involve definition of the coordinate system (FHC, FKC, FMCC, FLCC for the femur and TAC, TKC, TLCC, TMCC for the tibia) in this study is less than $1 \mathrm{~mm}$ with the exception of the TKC inter-observer value being $1.8 \mathrm{~mm}$. The maximum intra- and inter-observer error for these landmarks is $2.1 \mathrm{~mm}$ with the exception of the TKC inter-observer error being $3.3 \mathrm{~mm}$. Given the distance between the centre of the ankle and the centre of the tibial plateau, the maximum angular error is only $0.34^{\circ}$. It can be concluded that the CT scan is a safe tool to define the coronal, sagittal and horizontal plane of the femur and the tibia.

The inter-observer ICC ranked higher than 0.98 for all landmarks. Of those landmarks that define rotation of the femur, the lateral epicondyle was least reproducible. In the pre-operative planning, the surgical transepicondylar axis is often considered an optimal reference for horizontal plane alignment of the femoral component $^{23,40}$. In the post-operative evaluation of component alignment, the epicondylar axis is the only remaining landmark for defining the rotational position of the femoral component. Wai Hung et al. ${ }^{31}$ compared the CT registration error of the epicondylar axis to anatomic dissection and observed a mean $2.4^{\circ}$ error with conventional CT versus a significantly higher error of $2.9^{\circ}$ when a three-dimensional reconstruction was used. This could be explained by the inferior quality of the reconstruction or by the fact that a third dimension is taken into account. Consequently, it is important to consider the split error along the three Cartesian 
coordinate axes. As the epicondylar axis serves as a reference in the horizontal plane, registration error of the lateral epicondyle will have the greatest impact along the sagittal (AP) axis of the femur. The mean intra- and inter-observer error for the lateral epicondyle is respectively 0.5 and $0.8 \mathrm{~mm}$ along this axis. The maximum values are respectively $1.3 \mathrm{~mm}$ and $1.8 \mathrm{~mm}$. It appears that most of the error for the lateral epicondyle is observed along the vertical axis (PD). Mean intra- and inter-observer values along this axis are respectively $1.3 \mathrm{~mm}$ and $3.3 \mathrm{~mm}$, and maximum values 4.2 $\mathrm{mm}$ and $8.7 \mathrm{~mm}$. This three dimensional analysis of error explains why the anatomical and surgical trans-epicondylar axis show little angular intra- and interobserver variability when projected on the horizontal plane of the femur (Table 2). The lowest intra- and inter-observer variability is observed for the femoral posterior condylar line (respectively $0.17^{\circ}$ and $0.78^{\circ}$ ). The highest intra- and inter-observer variability is found for the trochlear antero-posterior axis (respectively $1.35^{\circ}$ and $3.26^{\circ}$ ). This axis is the most difficult to define precisely on a CT scan and it is the only axis related to rotational alignment that exceeds the clinically accepted $3^{\circ}$ threshold for its maximum angular error. As such, it cannot be regarded a reliable landmark. At the level of the tibia, the three axes that define rotation are the posterior condylar line, the tibial transverse axis and the tibial tubercle axes. Of those, the tibial transverse axis shows the least intra- and inter-observer variability, respectively $1.44^{\circ}$ and $1.66^{\circ}$. This confirms the findings of Cobb et al. ${ }^{27}$, who first defined this axis as a reliable landmark for describing rotation of the tibia. Both the tubercle axis and the posterior condylar line have a maximum error exceeding $3^{\circ}$ and cannot be recommended as reliable landmarks.

In conclusion, this study demonstrates low intra- and inter-observer variability in the CT registration of landmarks that define the coordinate system of the femur and the 
tibia. In the femur, the horizontal plane projections of the posterior condylar line and the surgical and anatomical transepicondylar axis can be determined precisely on a CT scan, using the described methodology, and can be recommended as reliable landmarks. In addition, the posterior condylar line is a hard reference, easily located during surgery, allowing to bridge the gap between the CAT scan and real femoral geometry. In the tibia, the least variability is found in the tibial transverse axis. Further research is needed to determine how precise this axis can be reconstructed on the real tibial geometry during surgery.

\section{Acknowledgement}

The study was carried out in the European Centre for Knee Research, which is sponsored by Smith and Nephew. 


\section{References}

1. Chauhan SK, Scott RG, Breidahl W, Beaver RJ. Computer-assisted knee arthroplasty versus a conventional jig-based technique. A randomised, prospective trial. J Bone Joint Surg Br. 2004; 86: 372-377

2. Amiot LP, Poulin F. Computed tomography-based navigation for hip, knee, and spine surgery. Clin Orthop Relat Res. 2004: 77-86

3. Bathis H, Perlick L, Luring C, Kalteis T, Grifka J. [CT-based and CT-free navigation in knee prosthesis implantation. Results of a prospective study]. Unfallchirurg. 2003; 106: 935-940

4. Bellemans J, Vandenneucker H, Vanlauwe J. Robot-assisted total knee arthroplasty. Clin Orthop Relat Res. 2007; 464: 111-116

5. Jazrawi LM, Birdzell L, Kummer FJ, Di Cesare PE. The accuracy of computed tomography for determining femoral and tibial total knee arthroplasty component rotation. J Arthroplasty. 2000; 15: 761-766

6. Moro-oka TA, Hamai S, Miura H, Shimoto T, Higaki H, Fregly BJ, et al. Dynamic activity dependence of in vivo normal knee kinematics. J Orthop Res. 2008; $26: 428-434$

7. Nishihara S, Sugano N, Ikai M, Sasama T, Tamura Y, Tamura S, et al. Accuracy evaluation of a shape-based registration method for a computer navigation system for total knee arthroplasty. J Knee Surg. 2003; 16: 98-105

8. van der Linden-van der Zwaag HMJ, Valstar ER, van der Molen AJ, Nelissen RGHH. Transepicondylar axis accuracy in computer assisted knee surgery: A comparison of the CT-based measured axis versus the CAS-determined axis. Computer Aided Surgery. 2008; 13: 200 - 206 
9. Geiger F, Parsch D. Intraoperative assessment of femoral component rotational alignment in total knee arthroplasty. Archives of Orthopaedic and Trauma Surgery. 2008; 128: 267-270

10. Kinzel V, Scaddan M, Bradley B, Shakespeare D. Varus/valgus alignment of the femur in total knee arthroplasty. Can accuracy be improved by pre-operative CT scanning? Knee. 2004; 11: 197-201

11. Galaud B, Beaufils P, Michaut M, Abadie P, Fallet L, Boisrenoult P. Distal femoral torsion: comparison of CT scan and intra-operative navigation instruments during total knee arthroplasty. A report of 70 cases. Rev Chir Orthop Reparatrice Appar Mot. 2008; 94:573-579.

12. Berger RA, Crossett LS, Jacobs JJ, Rubash HE. Malrotation causing patellofemoral complications after total knee arthroplasty. Clin Orthop Relat Res. 1998: $144-153$

13. Akagi M, Matsusue Y, Mata T, Asada Y, Horiguchi M, Iida H, et al. Effect of rotational alignment on patellar tracking in total knee arthroplasty. Clin Orthop Relat Res. 1999: 155-163

14. Anouchi YS, Whiteside LA, Kaiser AD, Milliano MT. The effects of axial rotational alignment of the femoral component on knee stability and patellar tracking in total knee arthroplasty demonstrated on autopsy specimens. Clin Orthop Relat Res. 1993: 170-177

15. Hanada H, Whiteside LA, Steiger J, Dyer P, Naito M. Bone landmarks are more reliable than tensioned gaps in TKA component alignment. Clin Orthop Relat Res. 2007; 462: 137-142

16. Matsuda S, Miura H, Nagamine R, Urabe K, Hirata G, Iwamoto Y. Effect of femoral and tibial component position on patellar tracking following total knee 
arthroplasty: 10-year follow-up of Miller-Galante I knees. Am J Knee Surg. $2001 ; 14: 152-156$

17. Miller MC, Berger RA, Petrella AJ, Karmas A, Rubash HE. Optimizing femoral component rotation in total knee arthroplasty. Clin Orthop Relat Res. 2001: 3845

18. Mochizuki RM, Schurman DJ. Patellar complications following total knee arthroplasty. J Bone Joint Surg Am. 1979; 61: 879-883

19. Olcott CW, Scott RD. The Ranawat Award. Femoral component rotation during total knee arthroplasty. Clin Orthop Relat Res. 1999: 39-42

20. Rhoads DD, Noble PC, Reuben JD, Mahoney OM, Tullos HS. The effect of femoral component position on patellar tracking after total knee arthroplasty. Clin Orthop Relat Res. 1990: 43-51

21. Yoshii I, Whiteside LA, White SE, Milliano MT. Influence of prosthetic joint line position on knee kinematics and patellar position. J Arthroplasty. 1991; 6: $169-177$

22. Defrate LE, Papannagari R, Gill TJ, Moses JM, Pathare NP, Li G. The 6 degrees of freedom kinematics of the knee after anterior cruciate ligament deficiency: an in vivo imaging analysis. Am J Sports Med. 2006; 34: 1240-1246

23. Griffin FM, Math K, Scuderi GR, Insall JN, Poilvache PL. Anatomy of the epicondyles of the distal femur: MRI analysis of normal knees. The Journal of Arthroplasty. 2000; 15: 354-359

24. Oberst M, Bertsch C, Lahm A, Wuerstlin S, Holz U. Regression and correlation analysis of preoperative versus intraoperative assessment of axes during navigated total knee arthroplasty. Computer Aided Surgery. 2006; 11: 87 - 91 
25. Talbot S, Bartlett J. The anterior surface of the femur as a new landmark for femoral component rotation in total knee arthroplasty. Knee Surgery, Sports Traumatology, Arthroscopy. 2008; 16: 258-262

26. Yoshino N, Takai S, Ohtsuki Y, Hirasawa Y. Computed tomography measurement of the surgical and clinical transepicondylar axis of the distal femur in osteoarthritic knees. The Journal of Arthroplasty. 2001; 16: 493-497

27. Cobb JP, Dixon H, Dandachli W, Iranpour F. The anatomical tibial axis: reliable rotational orientation in knee replacement. J Bone Joint Surg Br. 2008; 90-B: $1032-1038$

28. Han H, Chang C, Seong S, Lee S, Lee M. Evaluation of anatomic references for tibial sagittal alignment in total knee arthroplasty. Knee Surgery, Sports Traumatology, Arthroscopy. 2008; 16: 373-377

29. Jerosch J, Peuker E, Philipps B, Filler T. Interindividual reproducibility in perioperative rotational alignment of femoral components in knee prosthetic surgery using the transepicondylar axis. Knee Surg Sports Traumatol Arthrosc. 2002; 10: 194-197

30. Nofrini L, Slomczykowski M, Iacono F, Marcacci M. Evaluation of Accuracy in Ankle Center Location for Tibial Mechanical Axis Identification. Journal of Investigative Surgery. 2004; 17: 23 - 29

31. Wai Hung CL, Wai Pan Y, Kwong Yuen C, Hon Bong L, Lei Sha LW, Ho Man SW. Interobserver and Intraobserver Error in Distal Femur Transepicondylar Axis Measurement with Computed Tomography. The Journal of Arthroplasty. In Press, Corrected Proof: 
32. Pérez-Pérez A, Alesan A, Roca L. Measurement error: Inter-and Intraobserver Variability. An Empiric Study. International Journal of Anthropology. 1990; 5: $129-135$

33. LaPrade RF, Engebretsen AH, Ly TV, Johansen S, Wentorf FA, Engebretsen L. The Anatomy of the Medial Part of the Knee. J Bone Joint Surg Am. 2007; 89: 2000-2010

34. Bland JM, Altman DG. Measurement error. BMJ. 1996; 313: 744

35. LaPrade RF, Ly TV, Wentorf FA, Engebretsen L. The Posterolateral Attachments of the Knee: A Qualitative and Quantitative Morphologic Analysis of the Fibular Collateral Ligament, Popliteus Tendon, Popliteofibular Ligament, and Lateral Gastrocnemius Tendon. Am J Sports Med. 2003; 31: 854-860

36. Robinson M, Eckhoff DG, Reinig KD, Bagur MM, Bach JM. Variability of landmark identification in total knee arthroplasty. Clin Orthop Relat Res. 2006; 442: $57-62$

37. Yau WP, Leung A, Liu KG, Yan CH, Wong LLS, Chiu KY. Interobserver and Intra-observer Errors in Obtaining Visually Selected Anatomical Landmarks During Registration Process in Non-Image-Based Navigation-Assisted Total Knee Arthroplasty. The Journal of Arthroplasty. 2007; 22: 1150-1161

38. Lombardi AV Jr, Berend KR, Adams JB. Patient-specific approach in total knee arthroplasty. Orthopedics. 2008;31:927-930

39. Jeffery RS, Morris RW, Denham RA. Coronal alignment after total knee replacement. J Bone Joint Surg Br. 1991; 73: 709-714

40. Jenny JY, Boeri C. Low reproducibility of the intra-operative measurement of the transepicondylar axis during total knee replacement. Acta Orthop Scand. 2004; 75: 74-77 
41. Kessler O, Lacatusu E, Sommers MB, Mayr E, Bottlang M. Malrotation in total knee arthroplasty: effect on tibial cortex strain captured by laser-based strain acquisition. Clin Biomech (Bristol, Avon). 2006; 21: 603-609

42. Ritter MA, Faris PM, Keating EM, Meding JB. Postoperative alignment of total knee replacement. Its effect on survival. Clin Orthop Relat Res. 1994: 153-156

43. Werner FW, Ayers DC, Maletsky LP, Rullkoetter PJ. The effect of valgus/varus malalignment on load distribution in total knee replacements. J Biomech. 2005; 38: $349-355$

44. Perrin N, Stindel E, Roux C. BoneMorphing versus freehand localization of anatomical landmarks: Consequences for the reproducibility of implant positioning in total knee arthroplasty. Computer Aided Surgery. 2005; 10: 301 309

45. Fuiko R, Kotten B, Zettl R, Ritschl P. [The accuracy of palpation from orientation points for the navigated implantation of knee prostheses]. Orthopade. $2004 ; 33: 338-343$

46. Siston RA, Goodman SB, Patel JJ, Delp SL, Giori NJ. The high variability of tibial rotational alignment in total knee arthroplasty. Clin Orthop Relat Res. 2006; 452: 65-69

47. Stoeckl B, Nogler M, Krismer M, Beimel C, Moctezuma de la Barrera J-L, Kessler O. Reliability of the Transepicondylar Axis as an Anatomical Landmark in Total Knee Arthroplasty. The Journal of Arthroplasty. 2006; 21: 878-882

48. Aglietti P, Sensi L, Cuomo P, Ciardullo A. Rotational position of femoral and tibial components in TKA using the femoral transepicondylar axis. Clin Orthop Relat Res. 2008; 466:2751-2755. 
Table 1: Intra- and inter-observer distances to the observed position to the mean position for all landmarks in 3D and split along the anatomical axes.

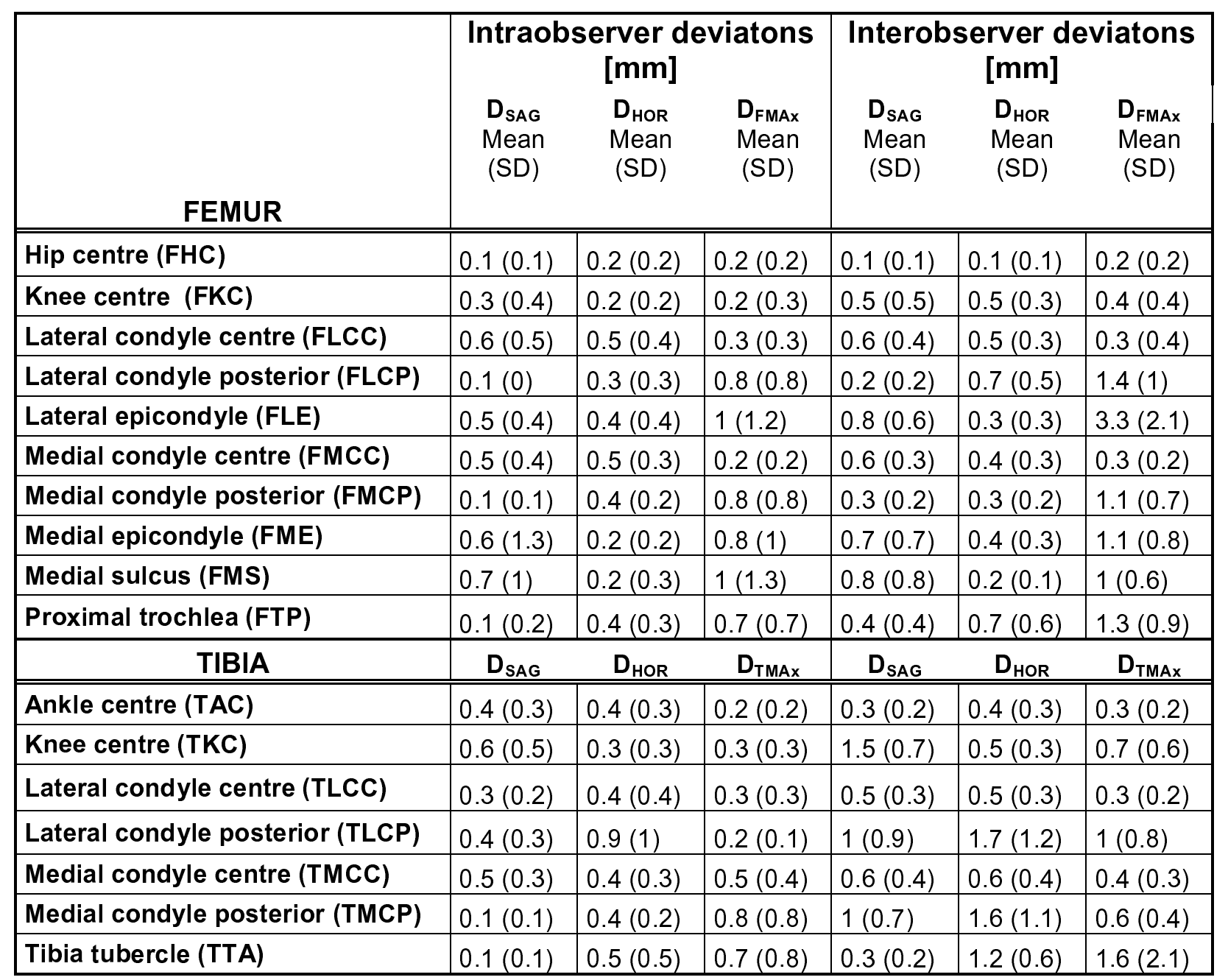


Table 2: Intra- and inter-observer variability of angular deviations of the femoral and tibial axes in the horizontal plane.

\begin{tabular}{|c|c|c|}
\hline Femur & $\begin{array}{c}\text { Intraobserver deviaton }\left[^{\circ}\right] \\
\text { Mean (Stdev) } \\
\text { Max }\end{array}$ & $\begin{array}{c}\text { Interobserver deviaton }\left[^{\circ}\right] \\
\text { Mean (Stdev) } \\
\text { Max }\end{array}$ \\
\hline Mechanical axis (FMAx) & $\begin{array}{c}0.05(0.03) \\
0.12 \\
\end{array}$ & $\begin{array}{c}0.08(0.08) \\
0.19 \\
\end{array}$ \\
\hline Anatomical transepicondylar axis (Anat TEA) & $\begin{array}{c}0.48(0.16) \\
0.73\end{array}$ & $\begin{array}{c}0.99(0.56) \\
1.7\end{array}$ \\
\hline Surgical transepicondylar axis (Surg TEA) & $\begin{array}{c}0.61(0.12) \\
0.71\end{array}$ & $\begin{array}{c}1.07(0.32) \\
1.38\end{array}$ \\
\hline Posterior condylar line (FPCL) & $\begin{array}{c}0.14(0.02) \\
0.17\end{array}$ & $\begin{array}{c}0.56(0.21) \\
0.78\end{array}$ \\
\hline Femoral transverse axis (FTAx) & $\begin{array}{c}1.23(0.27) \\
1.43\end{array}$ & $\begin{array}{c}1.36(0.33) \\
1.77\end{array}$ \\
\hline Trochlear anteroposterior axis (FTrAx) & $\begin{array}{c}0.94(0.38) \\
1.35\end{array}$ & $\begin{array}{c}2.07(0.76) \\
3.26\end{array}$ \\
\hline \multicolumn{3}{|l|}{ Tibia } \\
\hline Mechanical axis (TMAx) & $\begin{array}{c}0.15(0.05) \\
0.2\end{array}$ & $\begin{array}{c}0.28(0.04) \\
0.34\end{array}$ \\
\hline Posterior condylar line (TPCL) & $\begin{array}{c}1.37(0.37) \\
1.78\end{array}$ & $\begin{array}{c}3.16(1.77) \\
6.26\end{array}$ \\
\hline Tibial transverse axis (TTAx) & $\begin{array}{c}1.44(0.30) \\
1.89 \\
\end{array}$ & $\begin{array}{c}1.66(0.34) \\
2.03 \\
\end{array}$ \\
\hline Tubercle axis (TTubAx) & $\begin{array}{c}1.09(0.46) \\
1.89\end{array}$ & $\begin{array}{c}2.42(0.86) \\
3.32\end{array}$ \\
\hline
\end{tabular}




\section{Figure captions}

Figure 1: Three-dimensional model of the distal femur in frontal and lateral view. Abbreviations of the relevant surface and alignment points are shown on the image. For the definitions, see text.

Figure 2: Three-dimensional model of the proximal tibia in frontal and lateral view. Abbreviations of the relevant surface and alignment points are shown on the image. For the definitions, see text.

Figure 3: Intra-observer variability in the registration of the landmarks on the tibia and the femur, shown as mean value, maximum value and standard deviation.

Figure 4: Inter-observer variability in the registration of the landmarks on the tibia and the femur, shown as mean value, maximum value and standard deviation.

Figure 5: Graphical representation of the accuracy of the registration of the important reference points and axes for defining rotation in the femur and the tibia. The dark area is a representation of the mean error (enlarged for better visualization, scale in the legend), the lighter grey area represents the mean error +1 standard deviation $(\mathrm{SD})$. 
Figure 1

Click here to download high resolution image

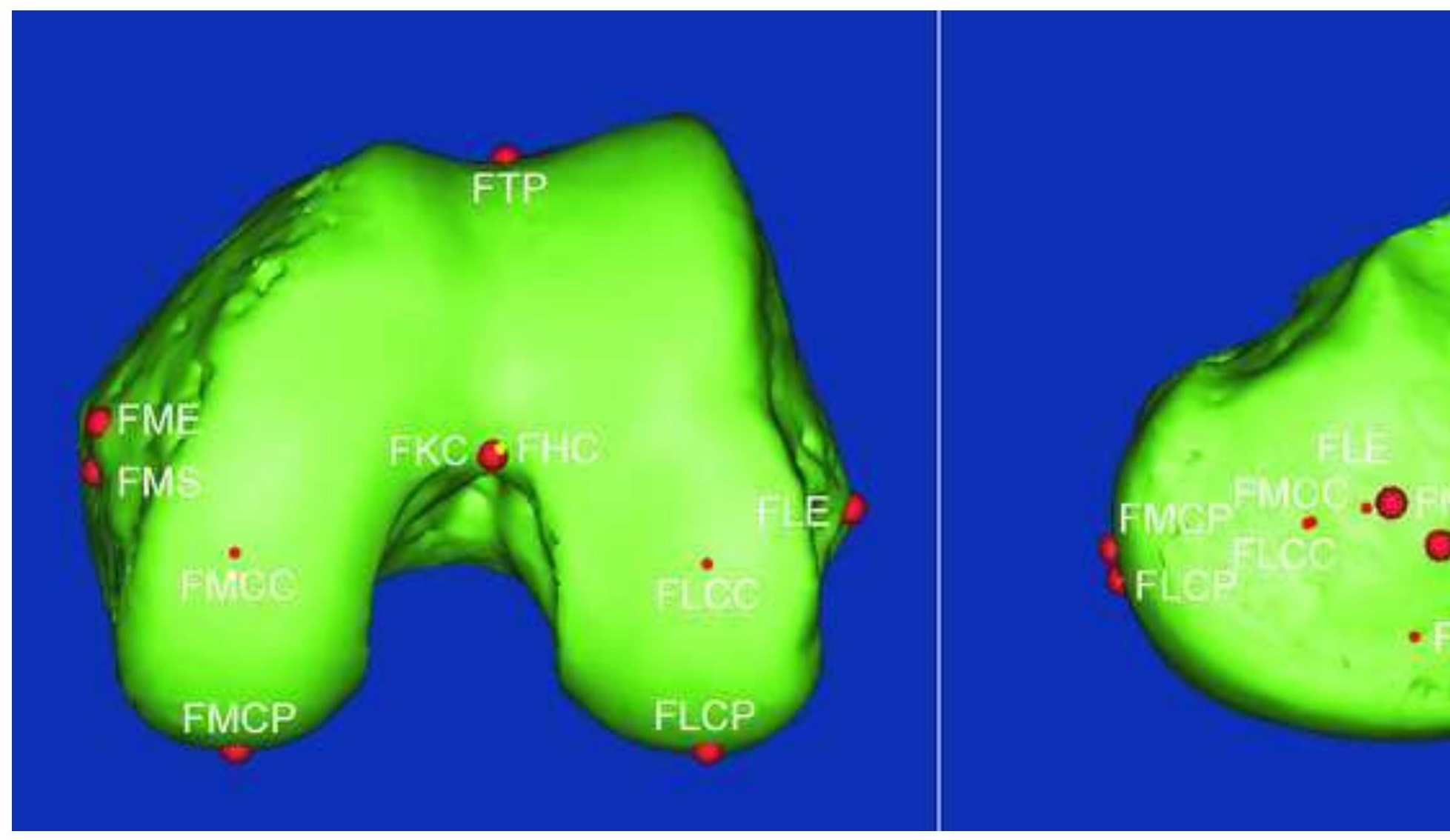


Figure 2

Click here to download high resolution image

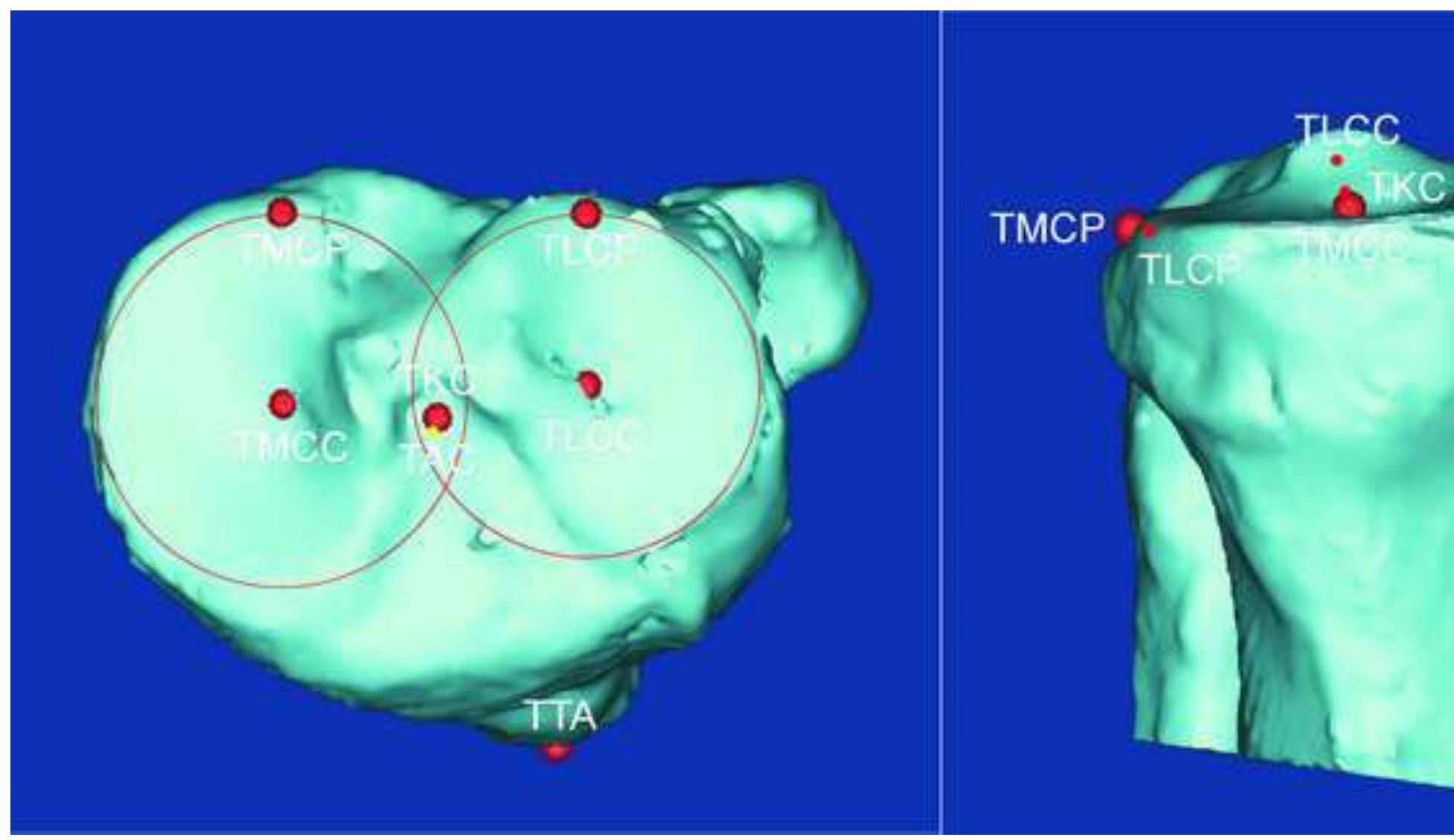


Figure 3

Click here to download high resolution image

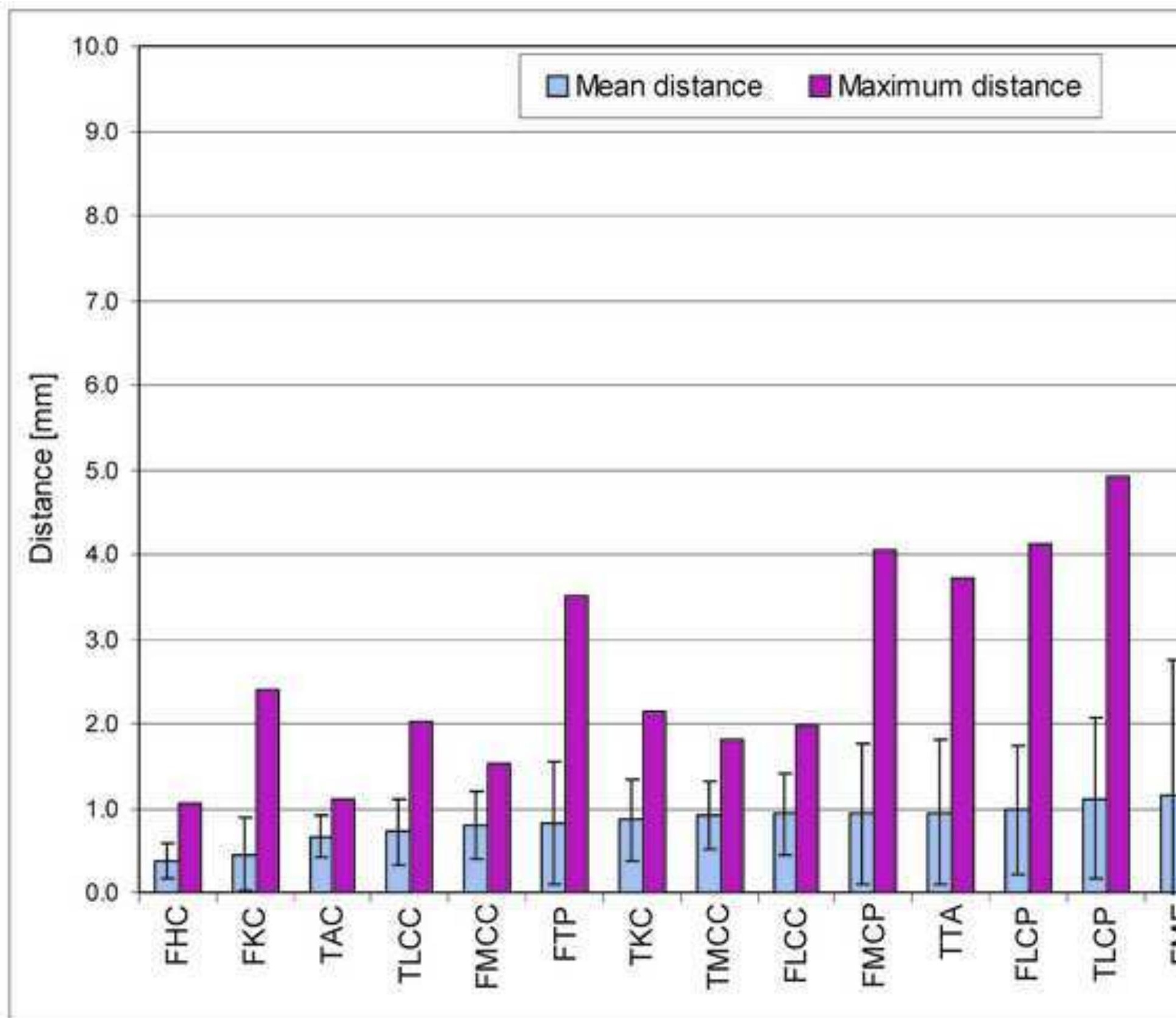




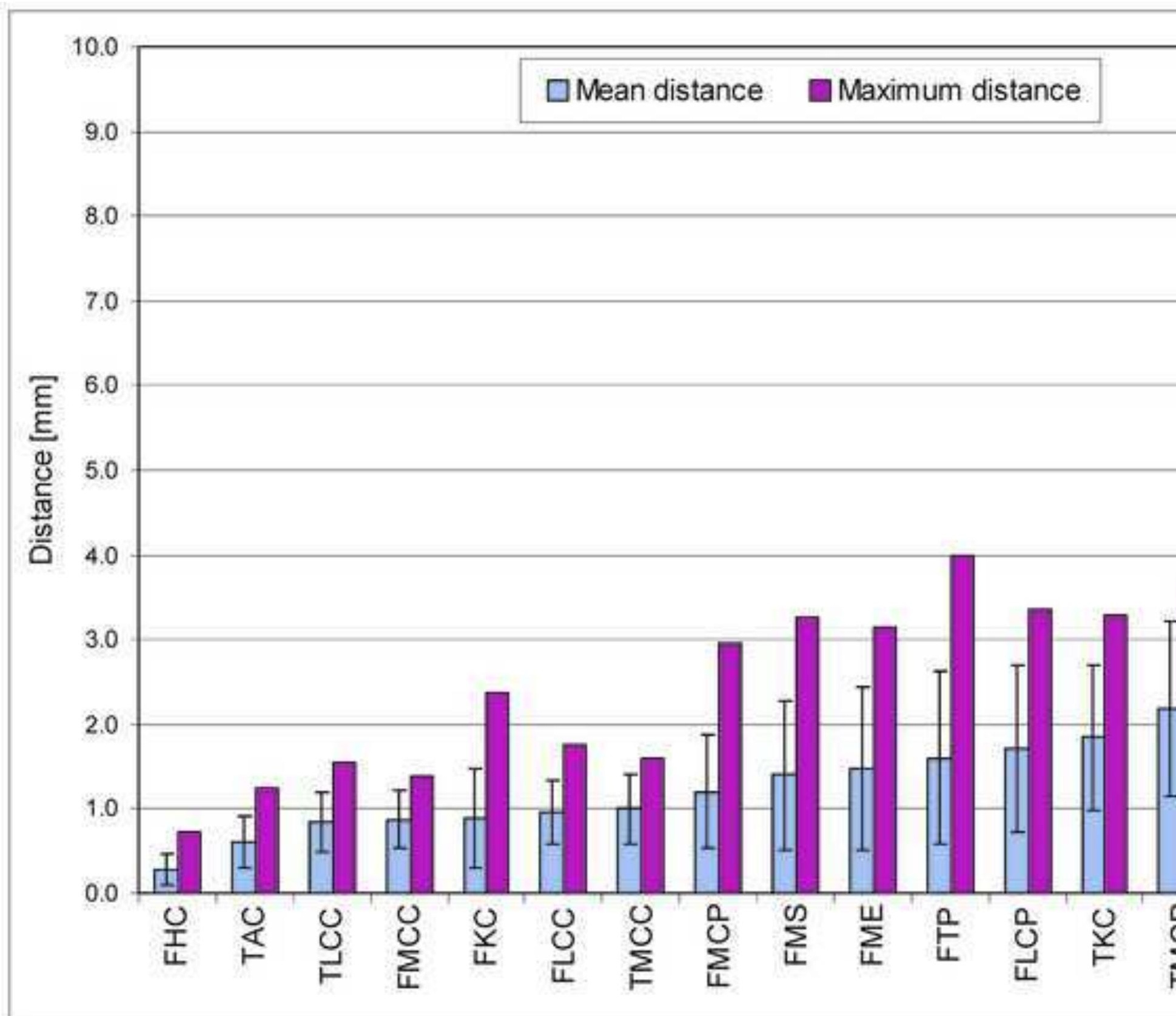


Figure 5

Click here to download high resolution image

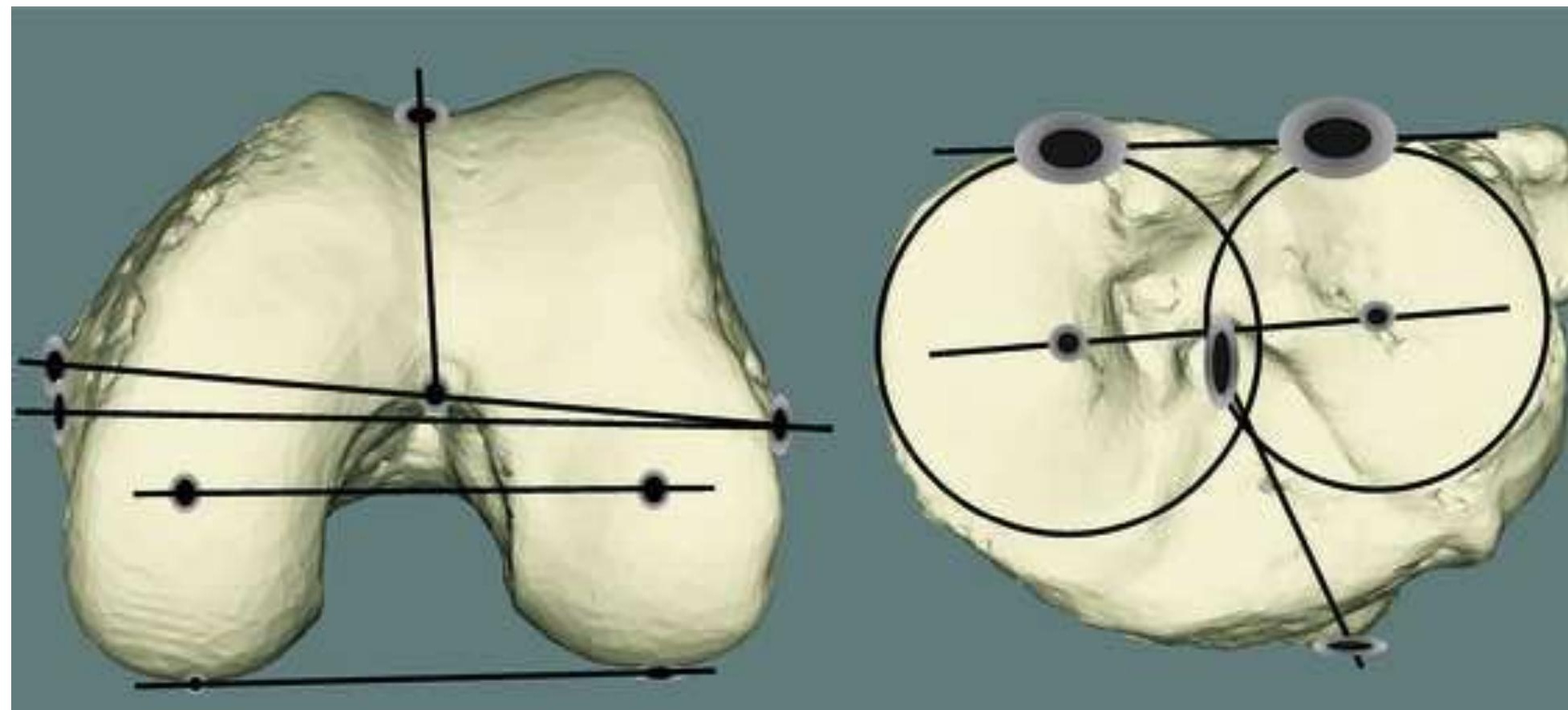

